\title{
Wege und Formen, Umwege und Umformungen: Quintus Smyrnaeus und die Rezeption der Trojasage in Kaiserzeit und Spätantike
}

\author{
KNUT USENER
}

Wolfgang Kullmann zum 80. Geburtstag

Für eine genauere räumliche oder zeitliche Einordnung der Posthomerica gibt es keinerlei sichere Anhaltspunkte: ${ }^{1}$ Im Horizont der Gattung überrascht es auch nicht, dass Bezugnahmen auf historische Gegebenheiten (vgl. etwa Q.S. 13.334349) oder Realien der Lebenswelt (vgl. etwa 6.531-536) nur höchst selten greifbar sind. Des Weiteren sind die Fragen, ob Quintus lateinische Literatur gekannt und verwendet habe, ${ }^{2}$ und wie sich das Verhältnis zwischen ihm und griechischen Autoren wie Oppian, Triphiodor oder Nonnos gestalte, ${ }^{3}$ nicht mit Sicherheit zu beantworten. Doch wenn man das geistig-poetologische Milieu der Posthomerica untersucht und in Beziehung zu anderen Dichtungen setzt, deren Entstehungszeit in etwa bekannt sind, wird man zwar kein für sich allein genommen verlässliches Kriterium erhalten, aber es können sich durch die Kombination verschiedener Aspekte sozusagen kumulative Evidenzen ergeben, die für eine Einordnung der Posthomerica in den literarischen Raum des späteren 3. Jh. n. Chr. sprechen.

Im Folgenden soll es daher um die Fragen gehen, wie der in größerem Rahmen betrachtete kulturelle und literarische Raum gestaltet ist, in dessen Bereich sich Quintus bewegt, und welche Einblicke in diesen Raum sein Werk erlaubt. Der in den Blick genommene Raum beherbergt generell griechische und römischlateinische Literatur der Kaiserzeit und der Spätantike. Doch soll hier ein Schwerpunkt auf die Werke einer dieser zwei Literaturen gelegt werden, nämlich auf die der Spätantike, insofern sie sich mit dem Trojanischen Sagenkreis (im Folgenden TSK) beschäftigt.

1 Wesentliches ist hierzu von Gärtner (2005) 23-26 gesagt. Vgl. weiter James (2004) XVII-XXI; Bär in diesem Band.

2 Vgl. hierzu insbesondere Gärtner (2005) 30-37 und passim zu Vergil; James in diesem Band.

3 Vgl. zu Oppian Kneebone in diesem Band; zu Triphiodor vgl. Dubielzig (1996) 8 und passim; zu Nonnos vgl. Shorrock in diesem Band. 
Den TSK haben insbesondere die Ilias, die Odyssee und der Epische Kyklos zum Thema. $\mathrm{Zu}$ Letzterem zählen im hier vorliegenden Zusammenhang die Kyprien, die Aethiopis, die Ilias mikra, die Iliupersis und die Nostoi ${ }^{4}$ Weitere Werke, die - wie etwa die in der Spätantike kaum bekannte Telegonie - in der Antike hinzugenommen wurden, bleiben hier unbeachtet. In den Posthomerica wird - das will der Titel andeuten - die Ilias, ebenso aber auch die Odyssee zunächst ausgespart. Doch wie Homer, so flicht auch Quintus zahlreiche Details aus dem Bereich der Vorgeschichte seines Erzählabschnitts in Form des integrierenden intertextuellen Verweises ein.

Dieser literarische Raum ist für den TSK recht elegant und vielfältig eingerichtet. Das Inventar stammt von verschiedenen Raumgestaltern, sozusagen von ,Fachbetrieben' und von ,Gelegenheitslieferanten " - oder anders formuliert: Wir haben zwischen Autoren mit thematischer und paradigmatischer Stoffverwendung zu differenzieren.

Mit thematischer Rezeption ist der Sachverhalt gemeint, dass ein Autor die Sage in Gänze oder in einem Teilaspekt ins Zentrum eines Werkes stellt, wie etwa Aischylos im Agamemnon, Sophokles im Aias oder auch Euripides in der Andromache.

Von paradigmatischer Rezeption kann man sprechen, wenn einzelne Szenen, Personen oder Motive in einem heterogenen Kontext - etwa im Zusammenhang philosophischer Gedankenentfaltung - aus ganz verschiedenen Gründen herangezogen werden. So ist etwa Xenophanes' Kritik an der homerischen (und hesiodeischen) Götterwelt eine paradigmatische Form der Rezeption, weil sie nur einen Aspekt eines noch dazu ganz bestimmten, nämlich des homerischen Epos herausgreift und in einen neuen Kontext ethisch-religiöser Prägung stellt. ${ }^{5}$

Bei den ,Fachbetrieben' und ,Gelegenheitslieferanten' können wir noch zwischen ,Direkt-“ und ,Zwischenhändlern“ unterscheiden, sodass wir von einer direkten, also unmittelbar auf Homers Epen zurückgreifenden, und einer indirekten, in der römischen Literatur insbesondere über Vergil umgeleiteten Bezugnahme sprechen können.

Homer und mit ihm der TSK durchdringen die gesamte antike Kultur: Nicht nur in enzyklopädischen Schriften, sondern selbst in speziellen Einzeldisziplinen wie etwa in geographischen und medizinischen Traktaten begegnen Hinweise oder sogar Zitate aus den homerischen Epen, da man in Homer alles Wissen der Welt angelegt fand und sich daher auf ihn als eine glaubwürdige Autorität glaubte beziehen zu dürfen. Die kaiserzeitliche und spätantike lateinische Literatur weist

4 Vgl. zur Zugehörigkeit dieser Epen zum Epischen Kyklos bereits das Scholion zu Clemens Alexandrinus, Protrepticus 2.30. Insgesamt vgl. Kullmann (1960) 18-28 und passim.

5 Vgl. u.a. Xenophanes 21 B 11 Diels / Kranz. 
(wie bereits die ihr vorangehende Literatur) alle Facetten der direkten und indirekten, thematischen und paradigmatischen Rezeption auf, insbesondere aber auch (dadurch unterschieden von der ihr vorangehenden Literatur) Bezugnahmen auf römische Autoren. ${ }^{6}$

Das Werk des Quintus vertritt den Bereich der thematischen und zugleich sowohl direkten als auch indirekten Rezeption, die den Aspekt derjenigen Geschehnisse in den Vordergrund stellt, die sich an Homers Ilias anschließen. Zugleich stellt es uns vor die Frage, woher die jeweiligen Informationen und Details stammen, die in den Posthomerica gestaltet sind. Neben Homers Epen kommen griechische, aber durchaus auch römische und lateinische Werke in Betracht. Schwierig ist die Frage, ob - und wenn ja: wie - Quintus Zugang zum Epischen Kyklos hatte. ${ }^{7}$

\section{Die Trojasage in der Überlieferung}

Die beiden Formen der thematischen und der paradigmatischen Rezeption dokumentieren die Beliebtheit der Trojasage, und es ist $\mathrm{zu}$ fragen, welche Szenen häufiger und detaillierter, welche seltener oder gar nicht oder nur andeutungsweise und ohne Details verwendet werden oder bekannt sind. Die Dokumente der Rezeption verfügen ferner stets auch über Potenziale, die sich in der späteren Literatur entfalten, den Stoff bereichern oder verkürzen, ihn variieren, umstrukturieren oder auch spielerisch abwandeln. So rezipiert also etwa Xenophanes eine speziell homerische Darstellung, den so genannten Götterapparat, und leitet aus der in seinen Augen unwürdigen Darstellung der göttlichen Handlungsweise eine poetologisch-theologische Schelte ab. ${ }^{8}$ Der Mythograph Fulgentius beispielsweise greift wiederum auf spezielle Details etwa der Ilias zurück, ${ }^{9}$ ohne den Handlungsoder Motivzusammenhang der jeweiligen Szene zu beachten: Er verfolgt im Horizont seiner speziellen allegorischen Mythendeutung sprachlich-hermeneutische Ziele, wenn er homerische Formulierungen zitiert.

Ohne im Detail auf die einzelnen Etappen der Überlieferung des TSK bei den Griechen eingehen zu können, ${ }^{10}$ sei diese nur so weit skizziert, wie dies für

6 Zur Differenzierung zwischen römischer (bis ins 2. Jh. n. Chr.) und lateinischer (über das 2. Jh. hinausgehender) Literatur vgl. Steinmetz (1982) 383 und passim; Fuhrmann (1999) 13-14.

7 Zur Diskussion vgl. u.a. Vian (1959a) 86-94; Gärtner (2005) 27-29 mit weiterer Literatur.

8 Vgl. etwa Xenophanes 21 B 11 Diels / Kranz

9 Vgl. etwa Fulg. myth. 3.1 mit Zitat von Il. 6.162 und 11.36 etc.

10 Allgemein zur Homerrezeption: Allen (1924); Mehmel (1954); Bonanno (1990); Lamberton / Keaney (1992); Richardson (1992a); Hölscher (1994). Zur frühgriechischen und klassischen Dichtung vgl. Davison (1955); Treu (1955); von Weber (1955); Rissman (1983); Davidson 
unseren Kontext notwendig scheint. Dabei muss neben der Bearbeitung einzelner Elemente des TSK insbesondere durch die Lyriker und Dramatiker darauf hingewiesen werden, dass es außer den homerischen Epen und den Epen des Kyklos eine weitere literarische Fixierung im Gattungshorizont des griechischen Epos nicht gegeben hat. Wichtig zu erwähnen ist allerdings, dass es bereits so genannte Trojaromane gegeben hat, die den Stoff in ungebundener Sprache und mit einer bisweilen geradezu unhomerischen Akzentuierung und Gestaltung neu geformt und verbreitet haben. Zu nennen sind hier Dictys und Dares - wobei man auch im letzteren Fall heute davon ausgeht, dass es tatsächlich eine griechische Schrift gegeben hat, aus der sich die spätantike lateinische Dares-Erzählung ableitet. ${ }^{11}$

Für die Überlieferung der Stoffgeschichte bis in die Spätantike, also bis in diejenige Epoche, die mit dem Jahr 284 beginnt und die bis ins erste Drittel des 7. Jh. (Tod von Mohamed 632; Isidor von Sevilla starb 636) oder sogar bis ins 8. Jh. (Beda Venerabilis, $\uparrow 735$ ) reicht, ${ }^{12}$ ist neben der griechischen auch die römische und lateinische Literatur bedeutsam - Letzteres allerdings unter der Prämisse, dass die lateinischsprachige Beschäftigung mit dem Mythos auch dort Leser fand, wo Griechisch die gängige Sprache war. Dass somit neben einer indirekten, über Zwischenstufen erfolgenden Rezeption auch - trotz des allmählichen Rückgangs der Griechischkenntnisse im lateinischsprachigen Reichsgebiet, der sich in ortsund zeitabhängiger Form und mit unterschiedlicher Intensität vollzogen hat - mit einem direkten Rückgriff speziell auf die Epen Homers zu rechnen ist, zeigen Beobachtungen zur Homerrezeption einzelner spätantiker Autoren, die hier nur in einer knappen Skizze angedeutet werden können. ${ }^{13}$ Für das Epos des Quintus bedeutet dies, dass auch lateinischsprachige Werke als Referenztexte in Betracht kommen können. Diese sind einer doppelten Brechung unterworfen: Sie stehen einerseits in der Tradition der griechischen (in diesem Falle natürlich insbesondere homerischen), zum anderen in der Tradition der römischen (oft besonders von Vergil geprägten) Literatur. Diese wiederum entfaltet eigene Charakteristika und beeinflusst den Strom der Stoffgeschichte des TSK auf ihre Weise.

(1987); Slings (1989). Zum Drama allgemein vgl. Lesky ( $\left.{ }^{3} 1972\right)$ 47-48 und passim; Latacz (1993) 96, 165, 269 und passim; zu Aischylos: Sideras (1971); zu Sophokles: Miller (1946) 99102. Zur Homerrezeption bei Platon vgl. Labarbe (1949); Lohse (1964; 1965; 1967). Zur Homerrezeption in der Historiographie vgl. Strasburger (1982). Zur Homerrezeption im Hellenismus vgl. Ludwich (1897); Skiadas (1965); Richardson (1992b); Rengakos (1993); Rengakos (1994). Zur Homerrezeption in der Kaiserzeit vgl. Buchheit (1956); Bouquiaux-Simon (1968); Glockmann (1968); Kindstrand (1973).

11 Vgl. hierzu etwa Merkle (1989); Beschorner (1992); Usener (1994).

12 Zum Ende der Spätantike mit dem Tod von Isidor vgl. Fuhrmann (1967); Döpp (1988); Gruber (1988).

13 Eine entsprechende Studie beabsichtigt der Verfasser demnächst vorzulegen, basierend auf seiner Habilitationsschrift. 
Grundlegend für die Rezeptionsgeschichte des TSK ist das im griechischen und römischen Bereich ähnlich konzipierte Schulwesen, ${ }^{14}$ in dessen Rahmen Schüler durch intensive Lektüre zentrale Autoren kennen lernen: So werden die Griechen insbesondere an Homer, die Römer zunächst an Ennius, später (seit Epirota) $)^{15}$ an Vergil, aber ebenso auch an Homer geschult, sofern sie Griechischunterricht erteilt bekommen. Wenn auch nicht die Werke komplett behandelt werden, so doch einzelne Bücher - bei Homer insbesondere die ersten zwei IliasBücher; die Odyssee ist offenbar nachgeordnet. Das Auswendiglernen ist immer wieder bis in byzantinische Zeit bezeugt und stellt somit ein wichtiges Instrumentarium für Dichter ebenso wie für Prosaschriftsteller zur Verfügung, die auf dieser Basis oft aus dem Kopf zitieren oder nachgestalten können. ${ }^{16}$

Für die Griechen kommt seit dem Hellenismus, für die Römer spätestens zu Beginn der Spätantike insbesondere auch noch die kommentierende und lexikalische Literatur hinzu: Auch sie ist eine wichtige Vermittlungsinstanz für Details des TSK.

Es gibt nahezu keinen griechischen Autor, der Homer gut, den weiteren Stoffbereich des TSK hingegen nicht kennt. Auch und gerade in der zweiten Sophistik spielen beide Zweige, der Homers mit den speziellen Epen der Ilias und der Odyssee und der mit anderen Dichtern verbundene übrige Sagenzusammenhang um Troja, eine große Rolle, und sei es, dass Homer lediglich als (stellvertretender) Referenzautor etwa bei Lukian, Philostrat oder Plutarch genannt wird. Homer kann (wenn man von den so genannten Chorizonten wie etwa Xenon und Hellanikos absieht) ohnehin synonym für die Ilias und die Odyssee stehen, aber darüber hinaus auch für den gesamten TSK. Über Details der Homerkenntnis sind wir insbesondere durch die ausführliche Studie über „Homer in der Zweiten Sophistik“ von Kindstrand informiert worden: ${ }^{17}$ An Dion von Prusa, Maximos von Tyros und Aelius Aristides zeigt Kindstrand exemplarisch auf, wie auf Homer zurückgegriffen wird: Homer als literarische Autorität, als Quelle von Zitaten und als Fundament griechischer Kultur. Dass bei der Beschäftigung mit Homer Texte als Grundlage verfügbar waren, zeigt Kindstrand anhand der Tatsache auf, dass bestimmte Lesarten der alexandrinischen Homerphilologen bei den Autoren der Zweiten Sophistik bekannt sind: Die literarische Arbeit geht klar

14 Dazu nach wie vor grundlegend, wenngleich in Details zu überprüfen Marrou ( $\left.{ }^{6} 1977\right)$. Vgl. ferner Morgan (1998), bes. 105-106 und passim.

$15 \mathrm{Zu}$ Quintus Caecilius Epirota, einem Freigelassenen des Atticus und Zeitgenossen des Gallus, vgl. Suet. Gram. 16: Epirota habe als erster Lehrer Vergilium et alios poetas novos in der Schule behandelt.

16 Michael Psellos (geboren 1018) etwa hat die gesamte Ilias auswendig gelernt (cf. Epitome 1.14).

17 Kindstrand (1973). Vgl. auch etwa Dubielzig (1996) 16-18 zu Triphiodor. 
über dasjenige Zitieren hinaus, das auf dem Auswendiglernen einzelner Passagen in der Schule beruht.

Anders hingegen liegt der Fall bei den Epen des Kyklos. Wenn Pausanias im 2. Jh. n. Chr. die Lektüre der Kyprien erwähnt (10.31.2), lässt sich dazu ein Papyrusfund (PRylands XXII), der Reste der Ilias mikra bietet, heranziehen. ${ }^{18}$ Doch bedeutet dieses griechische Zeugnis nichts für die Verfügbarkeit des KyprienTextes oder sogar des kompletten Kyklos im Westen des Reiches. Unter Severus Alexander (222-235) schließlich sollen die Werke des Kyklos insgesamt nicht mehr so hoch geschätzt worden und daher in Vergessenheit geraten sein - dies jedenfalls vermutet der spätantike, in Alexandria tätige Grammatiker Johannes Philoponos im 6. Jh., der offensichtlich keinen direkten Zugang zu diesen Epen mehr hatte. ${ }^{19}$

So stellt sich für uns die Frage, ob wir mit einiger Zuversicht feststellen können, welche dieser zahlreichen und recht unterschiedlichen Referenztexte Quintus verwendet: Hat er, einem Diogenes Laertius vergleichbar, zahlreiche Werke gelesen, in denen der Kyklos oder einzelne Elemente daraus bearbeitet wurden? Hat er den griechischen Dictys und den griechischen Dares gekannt? Diese beiden Prosafassungen bieten in unterschiedlichem Umfang und aus unterschiedlicher Perspektive einen Querschnitt durch den Troischen Sagenkreis. Oder hat Quintus Zugriff auf römische und lateinische Literatur gehabt?

Bei den Römern ist die Bekanntheit des TSK durch eine gleichsam gebrochene Überlieferung gegeben: Zunächst durch altlateinische Autoren - zu erwähnen sind insbesondere die Odusia des Livius Andronicus, aber auch Ennius und zahlreiche weitere Autoren. Deren Einfluss erlischt allmählich in dem Maße, in dem die Bekanntheit Vergils zunimmt, der die älteren Autoren geradezu verdrängt. Neben der Lektüre der homerischen Epen lernen die Römer den TSK also durch Vergil, bald aber auch durch andere Autoren wie Ovid oder Seneca kennen. ${ }^{20}$ Wichtig ist sodann neben Hygins Fabeln auch die Ilias Latina des Baebius (spätes 1. Jh. n. Chr.), die zusammen mit anderen Autoren für die mittelalterliche Trojarezeption Bedeutung erlangt. ${ }^{21}$

Neben den schon genannten und später noch knapp zu skizzierenden Pseudohistoriographen Dictys und Dares gibt es weitere Werke, die zumindest mit dem

18 Vgl. hierzu Severyns (1930).

19 Vgl. Johannes Philoponos, In Aristotelis Analytica posteriora commentaria 77 B 32, p. 156-157 Wallies.

20 Hier sollen nur literarische Quellen besprochen werden. Bildliche Darstellungen wie etwa die Tabulae Iliacae stellen eine weitere Möglichkeit der Vermittlung dar. Vgl. zu den Tabulae Iliacae Horsfall (1983) 144-147.

21 Einen knappen Überblick über die Homerrezeption in der römischen Literatur bietet Wlosok (1996); vgl. auch Vogt-Spira (1994). 
Inhalt, seltener mit speziellen Motiven des TSK vertraut machen: $\mathrm{Zu}$ erwähnen sind hier kleinere Periochae, wie sie etwa von Ps.-Ausonius erhalten sind, ${ }^{22}$ oder auch mythologische Handbücher, wie sie etwa von Fulgentius erhalten sind (Mythologicarum libri tres). ${ }^{23}$ Mit den Kenntnissen im Bereich des TSK sieht es somit in der Spätantike noch immer recht gut aus.

In Etappen eingeteilt, lässt sich die römisch-lateinische Seite der TSKRezeption in die Frühphase (Ennius etc.), in die Brechung durch Vergil und die augusteische sowie frühkaiserzeitliche Literatur und in die erneute Bearbeitung in der Spätantike (darüber später) gliedern. Die Spätantike baut dabei nicht nur auf der für sie vorbildlichen römischen und lateinischen Literatur auf, sondern greift auch auf die homerischen Epen direkt zurück. Anders sieht es mit den Epen des Kyklos aus: Die lateinische Spätantike bezieht - ähnlich der griechischen Literatur - ihre Kenntnisse über Details aus dem Bereich des Kyklos aus indirekt vermittelnden Texten.

Die Basis für die Auseinandersetzung mit dem TSK ist bei Griechen und Römern somit bis in die Spätantike unbezweifelbar gut, wenngleich teilweise aus unterschiedlichen Quellen gespeist und unterschiedlich gewichtet. Während die Griechen die Rezeption in der griechischen Sprache bevorzugen, lernen die Römer ,ihren“ TSK zunächst in griechischer Sprache, dann aber bald schon in römischer Brechung und $\mathrm{ab}$ dann oft und für lange Zeit parallel in beiden Sprachen kennen.

Die beiden ,Kriegstagebücher' von Dictys und Dares, Lexika sowie Handbücher könnten zumindest in ihrer griechischen Fassung zur Zeit des Quintus neben den Epen Homers Verbreitung gefunden haben: Sie sind alle als Referenztexte für die Posthomerica zu diskutieren.

Dies sind die Wege und Formen, Umwege und Umformungen des TSK bis in die Epoche des Quintus. Inwiefern auch bei den Griechen - speziell bei Quintus römische Texte die Stoffgestaltung beeinflussen, wird weiterhin zu untersuchen sein.

\section{Schulwesen und Kyklos}

Dass die Ilias und die Odyssee in antiken griechischen Schulen die allgemeine Textbasis in der Dichtung sind, steht außer Zweifel. Lukian, Philostrat oder auch

22 Vgl. die Ausonius-Ausgabe von Prete (1978), der die Periochae unter Nr. 28 abdruckt. Dazu ausführlich mit Literatur demnächst Usener (in der Habilitationsschrift).

23 Vgl. hierzu demnächst ausführlich Usener (in der Habilitationsschrift). 
Plutarch belegen die gute Vertrautheit in der Zweiten Sophistik, ${ }^{24}$ und im 4. Jh. kann man etwa auf Libanios oder Kaiser Julian hinweisen - sie alle reihen sich in die bis in die späte byzantinische Zeit hinein belegte Tradition guter Homerkenntnis ein. Die Texte der Ilias und der Odyssee sind zumindest in größeren Städten verfügbar. Anders sieht es, wie bereits angedeutet, mit den Epen des Kyklos aus: Deren Textverfügbarkeit ist eher problematisch.

Die Basis ist auch in der spätantiken lateinischen Literatur in einem insbesondere auf Homer (nicht aber auf Kyklische Epen) zurückgreifenden Griechischunterricht gegeben, der noch in dieser Epoche in vielen zumal größeren Städten bezeugt ist. Die Verbreitung der Texte muss immerhin so gut sein, dass es keine Probleme bereitet, an Unterrichtsmaterial zu kommen, und noch in der Spätantike bezeugt Ausonius für Gallien private Homerausgaben (Epistula 8.25 und Protrepticus 45-46). Homer wird auch außerhalb der Schule noch gelesen, ohne dass wesentliche Unterschiede zu heutigen Ausgaben zu verzeichnen sind - das erhellen etwa die Periochae-Zitate, Grammatikerzeugnisse oder auch Papyri.

Für Quintus bedeutet dies konkret, dass wir für den Hauptstoff der Posthomerica mit einer indirekten Überlieferung über verschiedene Autoren zu rechnen haben. Dass hierbei auch römische und lateinische Autoren in Betracht kommen, ist - ich betone dies nochmals nachdrücklich - durchaus diskutabel. Daher werde ich knapp skizzieren, wie sich der Troische Sagenkreis in der lateinischsprachigen Literatur speziell in der Kaiserzeit bis in die Spätantike hinein präsentiert.

\section{Römische und lateinische Literatur}

Die Odusia des Livius Andronicus, Ennius und weitere frühe Autoren geraten durch Vergils Aeneis bald ins Hintertreffen: Kenntnisse über den Troischen Sagenkreis bezieht man entweder ,direkt vom Hersteller', also zumeist Homer, oder über ,Zwischenhändler' wie Vergil, Ovid, Seneca, aber auch über Hygins Fabeln und die Ilias Latina des Baebius (spätes 1. Jh. n. Chr.), wobei Letztere neben Dictys und Dares für die mittelalterliche Trojarezeption besonders wichtig ist.

Somit bieten römische Texte zum einen rein stoffbezogene, zum anderen aber auch adaptierte und hermeneutisch geladene Vermittlungsfunktion.

Besonders erwähnenswert sind dabei die bereits genannten Verfasser bzw. Bearbeiter von Trojaromanen, Dictys und Dares.

24 Stellvertretend und zugleich grundlegend sei hier auf die Arbeit von Hillgruber (1994) 5-35 verwiesen, der zahlreiches Material und weitere Literatur nennt. 
In der auf einem griechischen Original aus dem 1./2. Jh. n. Chr. basierenden lateinischen Fassung der Ephemeris belli Troiani des Dictys Cretensis (wohl 4. Jh.) wird in sechs Büchern die gesamte Trojasage von der Vorgeschichte (Entführung Helenas, 1.3) bis zur Auswanderung der Aeneaden (5.17) und der Heimkehr der Griechen (6.1-15) in linearer Abfolge aus der fiktiven Perspektive und Innensicht eines Griechen mit bisweilen dramatischer Akzentuierung berichtet. ,Sachliche' Abweichungen von Homer sind keine Seltenheit. Die Ereignisse der Ilias nehmen zwar mehr Platz ein als die Geschehnisse der Odyssee (sowie der weiteren Heimkehrberichte), doch stehen sie nicht im Vordergrund. Wir haben es also hier wie auch bei Quintus mit einer anderen Akzentuierung als im homerischen Epos zu tun.

Die kleine, 44 Kapitel umfassende Prosa-Schrift Acta diurna des Dares Phrygius aus dem späten 5 . Jh., ${ }^{25}$ deren griechisches Original vielleicht aus dem frühen 3. Jh. stammt und daher als (Neben-)Quelle für Quintus in Frage kommt, kann als perspektivische Transformation zu Dictys gelesen werden: Dares berichtet - ebenso nur bisweilen in dramatischer Gestaltung - von der Kriegsursache bis hin zur Abreise der Griechen (42-43) und der Auswanderung einiger Troer (44), wobei er im Mäntelchen des Kriegsberichterstatters und Historikers auftritt und nicht konsequent aus trojanischer Sicht die Ereignisse schildert (vgl. bes. Kap. 12; 17; 37 und 44). Die Geschehnisse der Odyssee werden jedoch geradezu ausgeblendet. Das Ergebnis ist eine gewisse Entheroisierung der Hauptpersonen und eine Entmythologisierung der Stoffgestaltung. Eine Form der Homer-Epanorthose ist hier erkennbar, wie sie als geistige Strömung in dieser Zeit nicht singulär auftritt. $^{26}$

Neben Dictys und Dares gibt es die bereits genannten kleineren Periochae erhalten sind einige unter dem Namen des Ausonius. Die Tradition mythologischer Handbücher wie die des Hygin wird später von Fulgentius (im 5. / 6. Jh.) mit neuer Akzentuierung fortgesetzt.

Bei Fulgentius kann man zeigen, ${ }^{27}$ dass er kaum die Originaltexte verwendet, sondern dass er sich eher auf sekundäre Werke wie Handbücher, Kommentare oder Lexika stützt. Diese sind zwar heute nicht mehr erhalten, doch muss es sie bis in die Zeit des Fulgentius gegeben haben. Fulgentius kennt Homer eher als programmatischen Namen, hat aber von den mit ihm verbundenen Epen nicht oder nur in geringem Umfang Kenntnis.

25 Zu Dares' Schrift vgl. Beschorner (1992); zur Diskussion des Titels der Schrift vgl. Beschorner (1992) 191 mit weiterer Literatur.

26 Zur antiken Homerepanorthose vgl. Merkle (1989) 45-55 und passim; Beschorner (1992) 4 und passim.

27 Dazu demnächst ausführlich Usener (Habilitationsschrift). 
Lateinisch schreibende Autoren wie Ammian, Symmachus, die Autoren der Historia Augusta oder auch Dichter wie Claudian und Ausonius, Sidonius und Dracontius vermitteln uns einen guten Eindruck davon, wie unterschiedlich der Zugriff auf den TSK gestaltet werden kann: Neben einer gründlichen, beispielsweise durch Zitate oder gelehrte Anspielungen dokumentierten Kenntnis der homerischen Epen bei Ammian, Claudian und Ausonius gibt es die Bezugnahme auf Sagenelemente in Form der Brechung durch vermittelnde Autoren wie Vergil, Ovid und Seneca. Diese literarische Technik - die zitierende, anspielende oder im Horizont der jeweiligen kontextuellen und intentionalen Situation abwandelnde Bezugnahme - ist ein durchgängiges Charakteristikum in diesen spätantiken Texten. Bevorzugt wählen diese nun auch aus dem in Details divergierenden Fundus aus und gestalten somit die Sagen- und Motivkomplexe neu.

Weitere Schriften hat es gegeben, aus denen etwa auch spätantike Mediziner schöpfen können, wenn sie Homerverse heranziehen: So wird etwa im pseudoapuleischen Herbarius (CML 4, Kap. 48; 66.11) bei Beschwerden im Bereich der Gebärmutter das aus der Odyssee (10.305) bekannte $\mu \hat{\omega} \lambda v$ empfohlen, wobei wir wohl - aus literarischer Sicht - mit einer griechischen Vorlage zu rechnen haben.

Solange die Tradition detaillierter Kenntnisse bis in die Spätantike nachweisbar ist, kann man davon ausgehen, dass auch Quintus im Vorfeld oder sogar zu Beginn der Spätantike entsprechende Kenntnisse relativ leicht erwerben und wenn er sich denn belesen wollte - an geeignetes Material gelangen konnte.

\section{Die Trojasage in der Werkstatt: Einzelne Motive}

Im Folgenden werden drei Beispiele besprochen. Diese sind unter den Aspekten ausgewählt, dass erkennbar wird, wie Quintus die Durchführung des jeweiligen Themas 1) stofflich und 2) poetologisch im Horizont der Sagen- und Motivtradition gestaltet, und wie er insbesondere die Nähe oder die Distanz einerseits $\mathrm{zu}$ Homers Epen und andererseits zu lateinischen Werken insbesondere der Spätantike sucht.

Die Ermordung der Polyxena (Q.S. 14.214, 241, 313-319)

Polyxena, eine Tochter des Priamos, ist auf schicksalhafte und ausgesprochen dramatische Weise mit Achilleus verbunden, wird aber bei Homer (und entsprechend auch bei Baebius) nicht erwähnt. Wenn griechische und römischlateinische Autoren also Polyxena erwähnen, so nutzen und gestalten sie einen 
poetologischen Freiraum, der sie nicht an den Archegeten und - fast möchte man sagen - ,Überdichter' Homer bindet. Dies ist ein wesentlicher Grund dafür, dass das Verhältnis zwischen Polyxena und Achilleus auf ganz verschiedene Weisen erzählt und gewichtet wird.

Die Ermordung der Prinzessin ist stoffgeschichtlich erstmals für die Iliupersis bezeugt und wird von da an in der griechischen Literatur öfter erwähnt, etwa bei Ibykos, Sophokles oder auch Euripides. ${ }^{28}$ In der Iliupersis ist die vollständige Sage nur rekonstruierbar. ${ }^{29}$

Die Kombination der Motivelemente der Liebe, des in diesem besonderen Fall der Motivgestaltung durch sie bedingten Todes von Achilleus und der Opferung von Polyxena ist für uns erstmals bei Hygin (Fabula 110) greifbar. ${ }^{30}$ Fulgentius (Mythologiae 3.7) kennt die Sage von der todbringenden Liebe des Achilleus zu Polyxena, führt jedoch die Verletzung der Ferse als Todesursache an und kombiniert dadurch zwei verschiedene Sagenzüge. Die Liebschaft wird auf verschiedene Weisen erzählt.

28 Vgl. Iliupersis (Ilii excidium) arg. 22-23 Bernabé; Ibykos fr. 26 Page; Soph. (Polyxena) fr. 522528 Radt; Eur. Hec. 37-44; vgl. auch 218-224, 342-378, 508-582, sowie das Scholion zu Vers 41.

29 Wenn Polyxena am Grab des Achilleus ermordet werden soll, so wird man gerne mit der späteren Tradition annehmen, dass auch hier die Ursache in einer Liebesbeziehung zu suchen ist. Sehr wahrscheinlich war auch in der Iliupersis Polyxena die Geliebte des Helden, möglicherweise hat sie sogar mit dem feigen Mordanschlag auf ihn zu tun, dem Achilleus zum Opfer fällt: Er fällt in dieser Sagenversion nicht im Kampf, sondern wird aus dem Hinterhalt überfallen, als er sich unbewaffnet zu Hochzeitsverhandlungen in einen Hain vor Troja begibt. Die Ermordung von Polyxena am Grab des Achilleus ist eine Racheaktion, die den Totengeist des Helden besänftigen soll.

Die Liste der Autoren, die die dramatische Liebesbeziehung zwischen Achilleus und Polyxena bis hin zur grausamen Abschlachtung der Prinzessin (in welcher Form und mit welchen Motivvarianten auch immer) nachgestaltet haben, ist lang - bei den griechischen ebenso wie bei den römischen und lateinischen Autoren. In der römischen Literatur lässt sich das Motiv der Opferung der Polyxena nachweisen u.a. bei Verg. Aen. 3.321-324; Ov. Met. 13.439-532; Sen. Tro. 1132-1164; 1157ff. (vgl. zum Botenbericht von der Opferung der Polyxena den Kommentar von Fantham [1982] 375 zu Sen. Tro. 1118-1164, wo auch die Quellenfrage für Seneca diskutiert wird).

30 Ausonius, Epitaphium 26 scheint das Motiv ebenfalls zu kennen. Vgl. auch Ausonius, Technopaegnion 10.3. Das Motiv der Liebschaft kann jedoch bereits in der Iliupersis bekannt gewesen sein; Polyxena wird nur geopfert worden sein, weil sie als (vielleicht sogar verräterische) Geliebte im Zusammenhang mit dem Tod des Achilleus stand; möglicherweise ist also auch die Version von der Überwältigung des Achilleus im Apollonheiligtum im Kyklos vorbereitet. In den Kyprien wurde allerdings erzählt, dass Polyxena bei der Eroberung der Stadt durch eine Wunde stirbt, die ihr Odysseus und Diomedes beibringen; ihre Bestattung wird von Neoptolemos vollzogen; vgl. Cypria fr. 34 Bernabé. Vgl. insgesamt auch den Kommentar von Bömer (1982) 310-360 zu Ov. Met. 13.429-622. Das Scholion zu Stat. Ach. 1.134 nennt den Anschlag auf Achilleus im Zusammenhang mit dessen Liebe zu Polyxena, ohne die Opferung der Heroine zu erwähnen. 
Servius (zu Verg. Aen. 3.321) nennt zwei Versionen: (1) Achilleus verliebt sich in Polyxena, als er sie sieht, während er kämpft; (2) Achilleus verliebt sich in sie bei der Auslösung Hektors. Die dritte Variante findet sich bei Dictys (Ephem. 3.2) und Dares (De excid. 27): Achilleus erblickt Polyxena bei einer Opferhandlung.

Nach Servius (zu Verg. Aen. 3.321) wurde auch berichtet, dass sich Polyxena ihrerseits in Achilleus verliebt habe und daher den gewaltsamen Tod aequanimiter erduldet habe; von einem Plan, Achilleus zu ermorden, habe sie indes nichts gewusst. Dieser Version steht die Vorstellung der Polyxena als einer Verräterin gegenüber, die in der Spätantike bei Ausonius (Epitaphium 26), Dracontius (Orestis tragoedia 622ff.) und im anonymen Excidium Troie (12-13; 27) greifbar ist; sie liegt vielleicht bereits der Darstellung ihrer Opferung bei Ovid (Met. 13.439-532) zugrunde. Wenn sich Polyxena bei Dictys (Ephem. 3.24) dem Achilleus als Sklavin eius sponte anbietet, damit dieser ihren gefallenen Bruder Hektor auslöse, ${ }^{31}$ so ist ein Verliebtsein jedoch hier nicht auszuschließen.

Auch Quintus kennt Polyxena (Q.S. 14.214, 241, 315-319): Nach der Eroberung Trojas erscheint Achilleus seinem Sohn im Traum und fordert, nachdem er ihm allgemein gültige Lebensmaximen anempfohlen hat, dass er Agamemnon und Menelaos dazu veranlasse, Polyxena an seinem Grab zu opfern (14.185-222; Opferforderung: 209-222); wenn dies nicht geschehe, werde er einen Seesturm entfesseln, der ihnen zum Verhängnis würde. Neoptolemos beruft tags darauf eine Versammlung ein und richtet die Worte seines Vaters aus. Achilleus wird als ein Gott oder Dämon, der vormals unter Menschen weilte, verehrt und mit dem geforderten Opfer versöhnt, das von Hekabe beklagt wird (Klage der Hekabe: 14.272-301; Opfer: 14.313-319). Nach der Agora-Szene mit Neoptolemos lässt Poseidon das Meer von Stürmen aufwühlen, um der Forderung und Drohung des Achilleus Nachdruck zu verleihen (14.246-252). Polyxena wird nach ihrer Ermordung sofort freigegeben und darf von den Troern bestattet werden - sie wird zum Haus des Antenor gebracht, der sie seinem Sohn Eurymachos zur Braut bestimmt hatte (14.320-328).

Zuvor war bei Quintus von Polyxena nichts zu lesen: keine Liebesgeschichte, keine Andeutungen. Die (wohl auch hier im religiösen Kontext vollzogene) Ermordung erfolgt ohne vorherige oder spätere Legitimation etwa durch die Andeutung einer Liebesbeziehung, und sie erfolgt möglicherweise nur deshalb, weil es die Tradition so vorsieht. Nicht auszuschließen ist allerdings, dass hier ein Bild von Achilleus mit eingeblendet wird, wie wir es von Ovid (Met. 13.439-448) kennen: Ein Bild, in dem der Held als ein Dämon erscheint und die Opferung der

31 Bei Dracontius, Romulea 9.40-44 ist sie ebenso an der ,Lösung Hektors‘ beteiligt. Vgl. auch Patzig (1927/28). 
Polyxena fordert - dies würde das Fehlen der Legitimation oder Motivierung für die Opferung gleichsam ,kompensieren“, aber dennoch die Frage nach dem ,warum“ offen lassen - das Publikum muss sich die Antwort denken. Implizit ergibt sich aus der Einführung der Polyxena an dieser Stelle allerdings eine gewisse Dramatisierung: Nicht nur die Liebesbeziehung, sondern insbesondere ihre Beteiligung am Verrat lassen sich als Voraussetzung für Achilleus' Forderung zugrunde legen. Doch stirbt Achilleus bei Quintus nicht durch einen feigen Anschlag - wir haben es hier mit der Zusammenführung zweier heterogener Sagenzüge zu tun, die nicht ausgeglichen, sondern neu kombiniert werden. Zugleich wird Achilleus in dieser Szene durch die Worte, die er an seinen Sohn Neoptolemos richtet, als ein geradezu moralisierender Held gezeichnet - und somit entsteht ein gewisser Gegensatz zwischen dem fürsorglichen Vater einerseits und dem grausamen Totengeist andererseits: Auf der Ebene der poetologischen Gestaltung hat Quintus hier die innere Zerrissenheit des Helden, die die verschiedenen Ausformungen der Sage deutlich konturiert haben, in Form zweier disparater Einzelbilder nebeneinander gestellt - eine Technik, die insbesondere in Texten der Spätantike erkennbar ist.

Die soeben bei Quintus aufgezeigte Zusammenführung und neuartige Gestaltung heterogener Sagenversionen ist eine literarische Technik, wie wir sie insbesondere bei spätantiken Autoren antreffen. Musterhaft erkennbar ist diese Technik etwa bei Ausonius, Epitaphia heroum qui bello Troiano interfuerunt. Ausonius setzt die Erzählung vom Liebesverhältnis des Achilleus als bekannt voraus und gestaltet das Epigramm so, dass der Eindruck entsteht, die Liebe zu Polyxena werde von der Trojanerin nicht erwidert. ${ }^{32}$ Zugleich erlaubt die Neugestaltung einer Sagenversion einen Einblick in die poetologische Werkstatt des Dichters: Auf der Ebene nicht des Dargestellten, sondern des geistigen Konzeptes, das der Darstellung zugrunde liegt, lässt sich vermuten, dass sich Quintus in seiner Achilleus-Gestalt von Homer absetzen möchte. Quintus' Achilleus ist nicht mehr nur der ,homerische“ Krieger und der dämonische Totengeist, sondern gleichsam auch ein Mensch ,zum Anfassen'. Derartige poetologische Absichten lassen sich gut über die Profilierung des Personals und über die Handlungsführung mitteilen. Die Verbindlichkeit homerischer Gestaltung hat somit keine Gültigkeit mehr: Quintus tritt aus dem Schatten Homers dadurch heraus, dass er Parallelversionen zur Kenntnis nimmt und sie mit Homerischem zu Eigenem, zu Neuem verknüpft.

Für Quintus ist ein spezieller Referenztext von Hygin, Ausonius, Dictys, Dares oder auch Vergil nicht zu erweisen. Das Thema ist in der kaiserzeitlichen Literatur populär genug, so dass er das Rahmensujet sozusagen im Kopf gehabt haben und somit frei gestalten kann.

32 Aus. Epitaph. 26. 
Die Technik der eklektischen Kombination findet sich auch in anderem $\mathrm{Zu}$ sammenhang bei Quintus, wie wir im Beitrag von Ursula Gärtner erfahren (S. 211-240 in diesem Band): Quintus erweist sich hierin als ein Dichter im geistigen Umfeld spätantiker Literatur, in der gerne die Synthese verschiedener Strömungen mit dem Ziel versucht wird, neue Wege literarischen Gestaltens zu erproben.

\section{Die Schleifung Hektors (Q.S. 14.133)}

Jeder am antiken Mythos geschulte Mensch unserer Zeit, der von der Ermordung Hektors durch Achilleus gehört hat, verbindet damit sehr wahrscheinlich die Schleifung Hektors um die Mauern Trojas. Sie ist jedoch nicht in Homers Ilias oder Odyssee belegt, sondern erstmals in einem Drama auf der attischen Bühne (Euripides, Andromache 107-108) und sodann in der antiken Literatur mehrfach bezeugt: ${ }^{33}$ Homer lässt Achilleus den bereits erschlagenen Hektor an den Wagen binden und sofort - nicht über den ,Umweg' um die Mauern von Troja - zum Lager der Griechen schleifen (Il. 22.395-404). Erst später wird er ihn dann in rastloser und grausamer Wut um das Grabmal von Patroklos schleifen (Il. 24.12$18)$.

Die Schleifung Hektors um Troja ist auch bei Quintus (Q.S. 1.12; 14.133) belegt, dort allerdings jeweils katalogartig und somit ohne eine besondere szenische Dramatik. An einer Stelle jedoch (Q.S. 1.112) beklagt Andromache seine Schleifung um Troja in einer Klageszene, die zwar einerseits insofern ,homerisch ' ist, als Andromache dort das grausame Geschehen selbst mitansieht; doch zugleich unterscheidet sich Quintus von Homer eben durch das Detail der Schleifung ,um die Mauer'. Hektors Tod wird ohnehin nur in einer Rückblende à la Homer dargestellt, und wenn Quintus seine Schleifung erwähnt, dann reiht er sich einerseits in die Tradition des Euripides ein, hebt sich aber zugleich von anderen Darstellungen ab: Bei Quintus heißt es (14.133): „und wie er Hektor um die

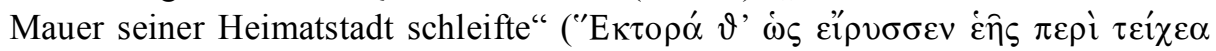
$\pi \alpha ́ \tau \rho \eta \varsigma)$ - ähnlich hatte dies auch Euripides gestaltet. Von einer dreimaligen Umrundung, wie sie etwa Vergil kennt, ist hier wie dort keine Rede. Damit ist zugleich die Raserei des Achilleus auf ein Maß reduziert, das eher der Darstellung des Helden bei Quintus entspricht. Quintus passt einen üblicherweise prominen-

33 In der römischen bzw. lateinischen Literatur sind u.a. zu nennen: Verg. Aen. 1.483-484 (vgl. 2.270-273 sowie den Kommentar von Austin [1964] bzw. [1971] jeweils zur Stelle); Ov. Met. 12.591; Baeb. Ilias 998; Hyg. Fab. 106; Dictys Ephem. 3.15 (dazu siehe S. 407). Hektors Tod durch die Schleifung ist erstmals bei Sophokles (Ajax 1029-1031) greifbar. Nach Ausonius (Periocha Iliadis 22) wird Hektor wie bei Vergil religatus ad currum ter circum moenia Troiana raptatur ,an den Streitwagen gebunden dreimal um die Mauern von Troja geschleift“. 
ten Sagenzug an die Konzeption ,seines‘ Heldenbildes an, das er von Achilleus entwirft. Damit eröffnet er erneut die Debatte um diesen griechischen Helden: Er zeichnet ihn als einen Menschen, der nicht übermäßig seinen Affekten nachgibt, sondern der sich - im Vergleich zum Helden Homers - beinahe schon gemäßigt verhält. Damit aber stellt sich Quintus in die Reihe derjenigen Autoren, die eine mehr oder weniger bewusste ,andere', jedenfalls nicht-homerische Darstellung der Handlung und der Charaktere entwickeln. Besonders weit gehen in dieser ,Disziplin` der so genannten Homer-Epanorthose Dictys und Dares.

Dictys (Ephem. 3.15) schildert die Schleifung zum Lager des Achilleus zwar wie zuvor Ennius in Anlehnung an die homerische Version, ${ }^{34}$ doch wird Hektor ex improviso, nicht im dramatischen Zweikampf, getötet. Dares (De excid. 24) erwähnt die Schleifung nicht. Die Verschiedenheit der Sagenaufbereitungen erlaubt es hier nicht, eine bestimmte Quelle für Quintus zu benennen.

Dictys und Dares entwerfen geradezu Gegenkonzeptionen zu Homer: Sie dokumentieren hierin ein hohes literarisches Selbstbewusstsein, wie es viele Autoren gerade in der Spätantike an den Tag legen: Dichter wie Claudian etwa sind sich ihrer Bedeutung und künstlerischen Fähigkeiten, die sich insbesondere im freien und geschickten Umgang mit verschiedenen Versionen einer und derselben Sage erweisen, sehr bewusst. ${ }^{35}$

\section{Die Rettung Nestors durch Antilochos (Q.S. 2.244-276)}

Nestors ältester Sohn Antilochos ist einer der so genannten ,kleineren Helden“ des TSK. Sein Schicksal verbindet ihn mit Memnon, durch den er im Kampf fällt: Näheres berichtet die Odyssee (4.188) freilich nicht - nur, dass Antilochos seinem (allerdings durch Paris) in Bedrängnis geratenen Vater hilft. ${ }^{36}$ In der Aethiopis war die Szene gestaltet (vgl. Aethiopis arg. 12 Bernabé), doch kennen wir keine weiteren Details. Dass sich Antilochos geradezu für seinen Vater opfert, erzählt - für uns erstmals greifbar - Pindar (Pythie 6.28-30). Diese dramatische Gestaltung wirkt weiter und wird etwa im pseudo-aristotelischen Peplos aufgegriffen, der wiederum Grundlage für Ausonius' Epitaphium 7 ist: In der lateinischen Literatur wird nur hier Antilochos' Tod als Selbstopferung für Nestor als Zeichen der virtus und der pietas (vgl. Vers 3) gedeutet. ${ }^{37}$

34 Enn. scen. 100-101 Vahlen (= Cic. Tusc. 1.105).

35 Zu Claudians Selbstbewusstsein vgl. u.a. Cameron (1970) 404 und passim.

36 Vgl. Od. 3.111-112 und 4.187-188; Aethiopis arg. 12-13 Bernabé; Pi. P. 6.28-30.

37 Aus. Epitaph. 7.3-4 (praemia virtutis similet pietatis adeptus, / servato Antilochus Nestore patre obii) bietet in etwa eine in die Ich-Perspektive umgesetzte Übertragung des pseudo-aristotelischen $\pi \dot{\varepsilon} \pi \lambda \mathrm{o}$-Gedichtes. 
Quintus reduziert nun geradezu den dramatischen Charakter des Opfertodes (Q.S. 2.244-276): Antilochos unterliegt in einem von ihm gegen Memnon geführten Kampf vor den Augen des Vaters, ohne sich explizit zu opfern. Memnon hatte Nestor angegriffen, und in diesen Kampf greift Antilochos ein, verletzt Memnon und stirbt letztlich durch ihn. Die dramatische Prägnanz der Szene, wie sie z.B. Pindar bietet, geht somit verloren. ${ }^{38}$ An einer anderen Stelle greift Quintus auf die Szene zurück: In 3.210 bleibt Nestor in der Trauer der Griechen um Antilochos gefasst, wohingegen sich Achilleus zur Rache rüstet. Wenig später $(3.517)^{39}$ rät Nestor nach dem Tod des Achilleus sogar in fast philosophischer Haltung zu maßvoller Trauer. Eine exakte Quelle ist auch hier nicht greifbar: Quintus verfügt offenbar über ein breit gestreutes, nicht aber auf immer genau bestimmbare Vorlagen zurückzuführendes Wissen um Details des TSK, die er nur aus der Lektüre ganz verschiedener Werke beziehen und mit denen er recht frei verfahren kann. Dies erweist ihn als eine Persönlichkeit im Kulturraum der Spätantike, in der umfassende Bildung in höheren Kreisen noch möglich und für viele erstrebenswert ist. Zugleich aber passt die Tendenz, hier wie auch etwa in der genannten Schleifung Hektors die Dramatisierung neu zu skalieren, in die kaiserzeitlichspätantike Literatur, in der Dramatisierung, Psychologisierung und Heroisierung bzw. Entheroisierung des Mythos die bevorzugten Kategorien sind, in denen der Mythos dem zeitgenössischen Lebensgefühl neu zugängig gemacht wird.

\section{Schluss}

Die vorgelegte Skizze erlaubt noch keine Auswertung oder Beantwortung etwa der Frage nach den Referenztexten oder Intentionen des Quintus: Deutlich wird, dass wir heute einen Dichter wie Quintus als Glied einer Kette von Autoren deuten sollten, die in ihrer Beschäftigung mit dem Mythos eine Fülle von Variationen zu einzelnen Szenen und Motiven vorfinden, aus der sie bewusst auswählen und in die sie durch ihre Auswahl gezielt eingreifen, um eigene poetologische Konzepte zu verfolgen und so den Faden der Sagentradierung weiter zu spinnen. Quintus huldigt nicht dem ,Überdichter' Homer, sondern er tritt ihm mit eigenen Akzentuierungen selbstbewusst zur Seite. Unser Kriterium wird nicht so sehr eine qualitative Bewertung (,besser' oder, schlechter') sein als vielmehr die Frage nach der Einordnung und Selbsteingliederung in den Strom der Überlieferung

38 Eine motivische Parallele, die jedoch durch neue Sinngebung spezifisch Geschehen und Handlung umdeutet, liegt bei Vergil vor (Aen. 10.769-820), wo Lausus seinem Vater Mezentius gegen Aeneas zu Hilfe kommt und schließlich stirbt. Zur Herkunft der Szene aus der Aethiopis vgl. Fraenkel (1932).

39 Vgl. ebenso Nestors Einstellung und Rat in Q.S. 5.605 nach dem Tod des Aias. 
einerseits und in den geistigen Kulturraum zur Zeit der Entstehung der Posthomerica andererseits. Beides ist in verschiedensten Brechungen greifbar, und wir sehen, dass es nicht nur ,den Homer', sondern darüber hinaus zahlreiche weitere Darstellungen gibt, die dadurch ,anders' sind, dass sie andere Intentionen verfolgen und anderen Prinzipien in Handlungsfügung und Stilisierung verpflichtet sind. Insofern sind alle Versionen ,gleichberechtigt', und wir lernen aus ihnen mehr über die jeweilige geistige Kultur, wenn wir sie nicht erst beurteilen oder abqualifizieren, sondern als das nehmen, was sie sind und sein wollen - Literatur zur Unterhaltung, zur Bildung, zur Konservierung - was immer das Genre ist, zu dem sich ein Werk bekennt.

Einige Beobachtungen in den Posthomerica sprechen dafür, dass Quintus in den kulturellen Raum der beginnenden Spätantike, sagen wir also in das eher späte 3. Jh. hineinragt. $\mathrm{Zu}$ diesen Beobachtungen gehört insbesondere eine in der Spätantike auftretende Vorliebe für die eklektische, sich in Form von Einzelbildern präsentierende Zusammenführung von verschiedenen Traditionen. Doch gehören auch Motivgestaltungen und Personenkonzepte dazu, die an die zeitgenössischen Lebensgefühle angepasst werden, und ferner einzelne Erscheinungen wie etwa die Rolle und Funktion des Schicksals ${ }^{40}$ als einer wesentlichen Instanz poetischer Aussage.

40 Vgl. hierzu den Beitrag von Gärtner in diesem Band. 
